\title{
Breakdown of frustrated absorption in $x$-ray sequential multiphoton ionization
}

\author{
Sang-Kil Son (손상길) $\odot,{ }^{1,2, *}$ Rebecca Boll $\odot,{ }^{3, \dagger}$ and Robin Santra $\oplus^{1,2,4, \ddagger}$ \\ ${ }^{1}$ Center for Free-Electron Laser Science, DESY, Notkestrasse 85, 22607 Hamburg, Germany \\ ${ }^{2}$ The Hamburg Centre for Ultrafast Imaging, Luruper Chaussee 149, 22761 Hamburg, Germany \\ ${ }^{3}$ European XFEL GmbH, Holzkoppel 4, 22869 Schenefeld, Germany \\ ${ }^{4}$ Department of Physics, Universität Hamburg, Jungiusstrasse 9, 20355 Hamburg, Germany
}

(Received 18 September 2019; revised manuscript received 20 January 2020; accepted 27 March 2020; published 20 April 2020)

\begin{abstract}
We investigate the frustrated absorption phenomenon of atomic systems driven by x-ray pulses of extremely high intensity. When an atom is exposed to intense $\mathrm{x}$-ray pulses generated by $\mathrm{x}$-ray free-electron lasers (XFELs), it undergoes complex ionization dynamics characterized by sequential multiphoton multiple ionization. Counterintuitively, as the pulse duration becomes shorter so that the intensity increases, the ionization becomes suppressed because of hollow-atom formation and the reduction of cross section. This is called frustrated absorption. However, as we report here, the paradigm of frustrated absorption can break down at extremely high intensity. By using a state-of-the-art theoretical tool, we examine the pulse-duration dependence of x-ray multiphoton ionization dynamics of heavy atoms, revealing that the reduced ionization for shorter pulses is due to the suppression of Auger decays, rather than the frustration of photoabsorption. Moreover, we predict a situation where ionization is, in fact, enhanced as the pulse duration is decreased and explain the mechanism why this happens. The present results demonstrate that the breakdown of frustrated absorption will emerge at the highest fluence currently available at XFEL facilities and will play an important role when terawatt-attosecond x-ray pulses come into realization.
\end{abstract}

DOI: 10.1103/PhysRevResearch.2.023053

\section{INTRODUCTION}

The advent of x-ray free-electron lasers (XFELs) [1-5], which provide ultrafast $\mathrm{X}$-ray pulses of unprecedentedly high intensity, has revolutionized various areas, covering atomic and molecular physics, solid-state physics, photochemistry, materials science, and structural biology [6-10]. Understanding the interaction of ultraintense and ultrashort x-ray pulses with atoms [11-15] and molecules [16-23] is a key ingredient for successful experiments with XFELs. In the optical regime, the ionization process induced by intense laser fields is described by nonsequential multiphoton ionization, where many photons must be absorbed simultaneously to ionize a valence electron and the perturbative rate of $n$-photon ionization is proportional to the $n$th power of the intensity [24]. In the $\mathrm{X}$-ray regime, however, the light-matter interaction is dominated by individual one-photon ionization events because the photon energy is sufficiently large to ionize a core electron. With high-intensity x-ray fields offered by XFELs, many one-photon ionizations occur sequentially, namely sequential

\footnotetext{
*sangkil.son@cfel.de

†rebecca.boll@xfel.eu

${ }^{\ddagger}$ robin.santra@cfel.de
}

Published by the American Physical Society under the terms of the Creative Commons Attribution 4.0 International license. Further distribution of this work must maintain attribution to the author(s) and the published article's title, journal citation, and DOI. single-photon single-electron interactions or sequential multiphoton multiple ionization $[11,25]$.

One of the unique features of this sequential XFEL-matter interaction is the phenomenon of frustrated absorption [16], where the degree of ionization by $\mathrm{x}$ rays is reduced as the pulse duration becomes close to or shorter than lifetimes of relaxation processes. This phenomenon is counterintuitive, because in this case higher intensity (shorter pulse) results in less ionization, which is the opposite of the behavior of conventional multiphoton ionization. The mechanism of frustrated absorption is based on hollow-atom formation [11]. Hollow atoms, which were originally found in collisions of highly charged ions with solid surfaces [26], have empty inner shells, so that the total $\mathrm{x}$-ray absorption cross section, which is dominated by innershell electrons, is reduced. Since the system becomes more transparent to $\mathrm{x}$ rays as intensity is increased, this phenomenon is also called intensity-induced $\mathrm{X}$-ray transparency [11]. This is particularly appealing for $\mathrm{x}$ ray crystallography [27] and diffractive imaging [28], because it indicates a way to reduce electronic radiation damage (i.e., the ionization degree) by using ultrashort pulses [29]. It is also important to understand the dynamical behavior of atomic ionization in the formation of warm or hot dense matter by using XFELs [30]. Frustrated absorption in x-ray sequential multiphoton ionization was discovered in pioneering soft- $\mathrm{X}$ ray XFEL experiments on systems containing light atoms $[11,16]$, but it has not yet been verified for heavy atoms and hard $\mathrm{x}$ rays.

In the sequential ionization model, a succession of ionic charge states are formed as multiple ionization proceeds and 
the ionization potential increases with increasing ionic charge. Beyond a certain charge state, the photon energy is insufficient to ionize further, so one-photon ionization stops, which defines the direct one-photon ionization limit [31]. In this limit, the final charge is determined by the last ionic state that-in its ground electronic state - can be ionized by one-photon ionization. Multiple resonant excitation combined with a broad energy bandwidth of XFEL pulses can overcome this limit, leading to so-called resonance-enabled or -enhanced $\mathrm{X}$-ray multiple ionization (REXMI) [13,32-34]. When a hard-X-ray photon interacts with deep innershell electrons of heavy atoms, it is necessary to take into account relativistic effects in the electronic structure $[14,35]$. A combination of resonant and relativistic effects escalates the complexity of the ionization dynamics [31].

With current XFEL technology, it is possible to achieve an $\mathrm{X}$-ray intensity on target approaching $10^{20} \mathrm{~W} / \mathrm{cm}^{2}$ [36]. Furthermore, cutting-edge techniques promise to shorten XFEL pulses into the attosecond regime [37-41]. To understand and design XFEL experiments for such extreme conditions, it is requisite to benchmark the pulse-duration dependence of ionization dynamics at ultrahigh fluences and/or ultrashort pulses.

In this paper, we investigate the pulse-duration dependence of ionization dynamics of $\mathrm{Xe}$ and $\mathrm{Ne}$ atoms at very high fluences, which are reachable with current XFEL facilities, over a broad range of pulse durations down to subfemtoseconds, which will be accessible in the near future. We revisit the frustrated-absorption phenomenon at such extreme conditions and report its breakdown, arguing that the frustratedabsorption picture is valid only at moderate fluences. Therefore our finding offers critical insight regarding XFEL-matter interactions at extreme conditions of significance for future XFEL experiments.

\section{THEORY}

We employ the XATOM toolkit $[42,43]$ to describe ionization dynamics of atoms, which has been tested for a series of gas-phase XFEL experiments [12-15,32,35]. It has been extended to incorporate both resonance and relativistic effects [31], which makes it suitable to explore ionization dynamics of heavy atoms over a wide range of x-ray pulse parameters in terms of photon energy, fluence, and pulse duration. The XATOM toolkit calculates photoabsorption cross sections, Auger and Coster-Kronig rates, and fluorescence rates for any given charges and electronic configurations that are formed during multiple ionization dynamics. Using a rate-equation approach $[11,25]$, it solves a set of coupled rate equations to describe the dynamical behavior of atoms during intense XFEL pulses.

First, we investigate multiphoton multiple ionization of Xe at $1200 \mathrm{eV}$, where ionization dynamics are mainly initiated by $M$-shell $(n=3)$ ionization. The number of coupled rate equations, which is equivalent to the number of electronic configurations that are formed by removing 0,1 , or more electrons from initially occupied subshells of Xe and placing them into $(n, l)$ subshells $(3 \leqslant n \leqslant 30$ and $0 \leqslant l \leqslant 7)$, is estimated to be $\sim 4.2 \times 10^{60}[15,34]$. To handle such a huge number of rate equations, we use the Monte Carlo on-the-fly scheme [14].

\section{RESULTS AND DISCUSSION}

\section{A. Pulse-duration dependence of ionization dynamics of Xe at $1200 \mathrm{eV}$}

Figure 1 shows the final mean charge of $\mathrm{Xe}$ at a photon energy of $1200 \mathrm{eV}$ for five different fluences. For three cases, the corresponding charge-state distributions are displayed in Appendix A. The temporal pulse shape assumed is Gaussian. The pulse duration (full width at half-maximum, FWHM) varies from 0.2 fs to $100 \mathrm{ps}$. The highest peak intensity produced by the highest fluence and the shortest pulse duration considered $\left(1200 \mathrm{eV}, 1 \times 10^{12} \mathrm{ph} / \mu \mathrm{m}^{2}\right.$, and $0.2 \mathrm{fs}$ FWHM) is $\sim 9 \times 10^{19} \mathrm{~W} / \mathrm{cm}^{2}$. A $1 \%$ spectral bandwidth $(\mathrm{FWHM})$ is used. Here, about 1000 Monte Carlo trajectories through the electronic configuration space are used for calculating an average for each pulse duration, and the statistical error for the mean charge is represented by the error bar. For the low-fluence cases $\left(5 \times 10^{10} \mathrm{ph} / \mu \mathrm{m}^{2}\right.$ and $\left.1 \times 10^{11} \mathrm{ph} / \mu \mathrm{m}^{2}\right)$, the mean charge decreases for shorter pulse durations, as predicted by the frustrated-absorption picture $[11,16]$. Surprisingly, one can see the opposite trend when the fluence is cranked up. For high fluences, the mean charge increases for shorter pulse durations. For the highest fluence $\left(1 \times 10^{12}\right.$ $\left.\mathrm{ph} / \mu \mathrm{m}^{2}\right)$, the mean charge is +26.7 for $100 \mathrm{ps}$, while it becomes +34.0 for $1 \mathrm{fs}$, which is clearly the opposite of the behavior of frustrated absorption. A first hint of such an effect was presented in Ref. [34] for resonance-enhanced ionization of $\mathrm{Kr}$.

To elucidate this phenomenon, we plot in Fig. 2 the number of $x$-ray-induced processes as a function of pulse duration for the lowest $\left(5 \times 10^{10} \mathrm{ph} / \mu \mathrm{m}^{2}\right)$ and highest $\left(1 \times 10^{12} \mathrm{ph} / \mu \mathrm{m}^{2}\right)$ fluences used in Fig. 1. In the low-fluence case (a), it is noticeable that the number of Auger decays decreases as the pulse duration becomes shorter, which is consistent with the observation in $[34,44,45]$. This is because Auger decays are beaten not only by $M$-shell photoabsorptions, but also by $N$ and $O$-shell photoabsorptions. The latter effectively reduce the number of outershell electrons available for Auger processes.

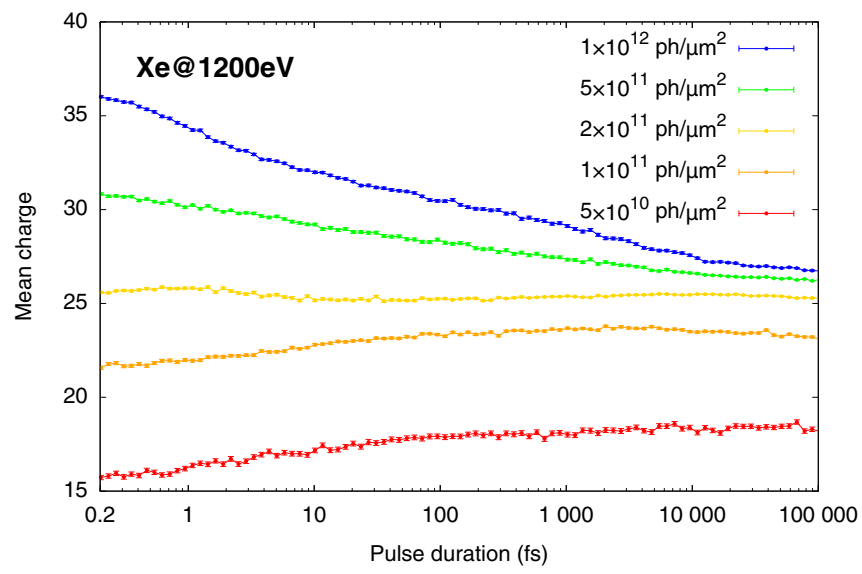

FIG. 1. Final mean charge of Xe at $1200 \mathrm{eV}$ as a function of pulse duration. The error bar indicates the statistical error. 

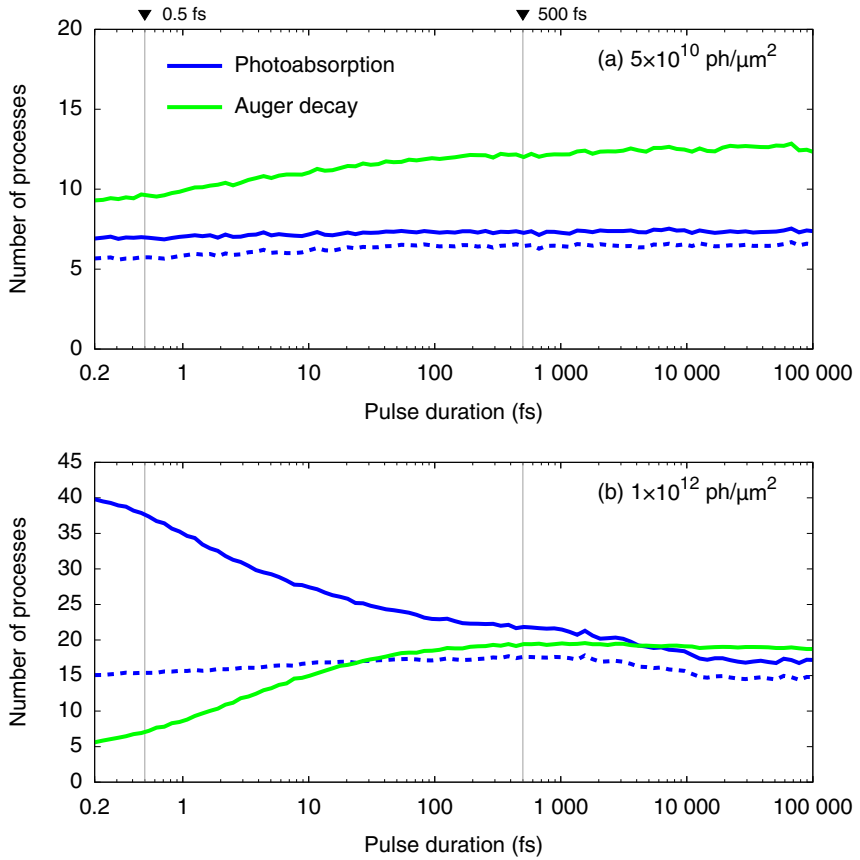

FIG. 2. Number of $\mathrm{x}$-ray-induced processes in $\mathrm{Xe}$ at $1200 \mathrm{eV}$ as a function of the pulse duration: (a) low fluence, $5 \times 10^{10} \mathrm{ph} / \mu \mathrm{m}^{2}$ and (b) high fluence, $1 \times 10^{12} \mathrm{ph} / \mu \mathrm{m}^{2}$. The solid blue line represents the total number of photons absorbed by photoionization and photoexcitation during a pulse, and the solid green line is the number of Auger decays occurring during and after the pulse. The $M$-shell contribution in the total number of photoabsorption events is plotted with the dashed blue line. The pulse durations of $0.5 \mathrm{fs}$ and $500 \mathrm{fs}$, which are used in Figs. 3 and 5, are marked with vertical lines.

See Appendix B for a detailed discussion of the competition between Auger and photoionization processes. It is interesting to see that the number of photoabsorptions, which is the total number of photons absorbed by ionizations and resonant excitations, is almost flat. It implies that photoabsorption is not actually frustrated; instead, the reduction of the mean charge for shorter pulses at $5 \times 10^{10} \mathrm{ph} / \mu \mathrm{m}^{2}$ in Fig. 1, which superficially exhibits the trend of frustrated absorption, turns out to be due to the reduced number of Auger decays. In the high-fluence case (b), the number of photoabsorptions rises dramatically and the number of Auger decays drops further as the pulse duration is shortened. The $M$-shell contribution to the total photoabsorption events (dashed blue line) is still practically flat, which will be discussed later. For a very short pulse, the photon flux becomes sufficiently high so that photoabsorption rates always beat Auger rates, as shown in Fig. 9(b).

The system can absorb several photons within an ultrashort time interval, before any relaxation process happens. We refer to this situation as "quasinonsequential" absorption in the $\mathrm{x}$-ray regime: multielectron ionization happens via real intermediate states step-by-step in a series of single-photon single ionization events, but at such high fluence, the intrinsic timescale between ionization steps, which is set by the inverse of the fastest single-photon ionization rate, becomes short in comparison to innershell lifetimes. X-ray quasinonsequential multiple ionization displays similarities to nonsequential
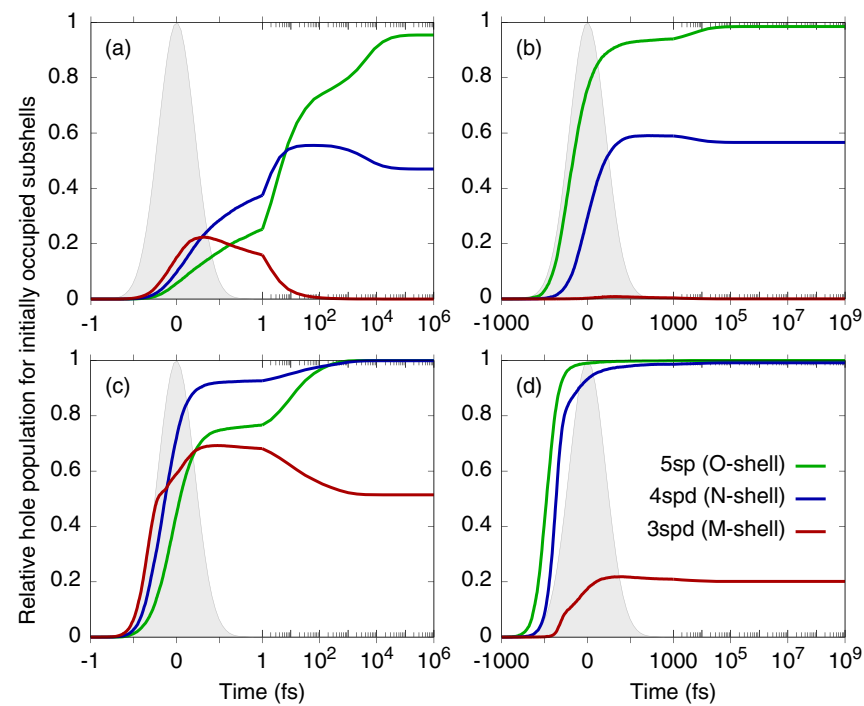

FIG. 3. Hole population dynamics of $\mathrm{Xe}$ at $1200 \mathrm{eV}$ for four different sets of pulse parameters: (a) $5 \times 10^{10} \mathrm{ph} / \mu \mathrm{m}^{2}$ and $0.5 \mathrm{fs}$ FWHM, (b) $5 \times 10^{10} \mathrm{ph} / \mu \mathrm{m}^{2}$ and 500 fs FWHM, (c) $1 \times$ $10^{12} \mathrm{ph} / \mu \mathrm{m}^{2}$ and $0.5 \mathrm{fs}$ FWHM, and (d) $1 \times 10^{12} \mathrm{ph} / \mu \mathrm{m}^{2}$ and $500 \mathrm{fs}$ FWHM. The solid lines are relative hole populations for the subshells that are initially occupied. The shaded gray areas show the temporal envelope of the Gaussian x-ray pulse for a given pulse duration. Up to the end of the pulse (defined as two times the pulse duration), the time axis is on a linear scale, whereas it is on a logarithmic scale after the pulse end.

ionization-more absorption for shorter pulses, as demonstrated in Fig. 2(b). However, this phenomenon differs from more conventional nonsequential multiphoton ionization processes in various aspects. In the optical regime, nonsequential (direct) multiphoton absorption induces single ionization via virtual states whose lifetime is given by the reciprocal of the detuning [46]. It is also different from nonsequential multiple ionization [47-49]. Note that in sequential x-ray multiphoton multiple ionization, the effective lifetime of an intermediate, innershell-excited state is determined by the natural relaxation lifetime of the innershell vacancy, whereas in the present x-ray quasinonsequential absorption the effective intermediate-state lifetime is dominated by photoabsorption.

Since $\mathrm{x}$-ray multiphoton ionization is dominantly initiated by core $(M$-shell) ionization, the quasinonsequential ionization naturally produces a multiple core-hole state. Thus, by inspecting the multiple hole dynamics during the $\mathrm{x}$-ray pulses, we will obtain a better understanding of the underlying ionization mechanism.

\section{B. Hole population dynamics of Xe at $1200 \mathrm{eV}$}

We show the hole population dynamics of Xe at $1200 \mathrm{eV}$ in Fig. 3. The time-dependent hole populations, averaged over 2000 Monte Carlo trajectories, are analyzed for different shells: $M$ (red line), $N$ (blue line), and $O$ (green line) shells and plotted as relative hole populations with respect to the initial occupation numbers corresponding to neutral Xe. The temporal pulse envelope is shown as a shaded gray area. To capture long-lived relaxation processes, the dynamics are 
computed up to $10^{6}$ times the pulse duration after the end of the pulse. For each panel in Fig. 3, the left half of the $x$ axis is shown on a linear scale, whereas the right half is on a logarithmic scale. Four different combinations of pulse parameters are chosen: low $\left(5 \times 10^{10} \mathrm{ph} / \mu \mathrm{m}^{2}\right) / \mathrm{high}$ $\left(1 \times 10^{12} \mathrm{ph} / \mu \mathrm{m}^{2}\right)$ fluence and short $(0.5 \mathrm{fs}) /$ long $(500 \mathrm{fs})$ pulse.

First, let us examine the low-fluence cases [(a) and (b)]. The main difference between short and long pulses is the different slopes of the hole populations for individual shells at the beginning of the pulse. For the long-pulse case (b), there is almost no population for $M$-shell holes, even though the $M$ shell has the highest photoabsorption cross section at $1200 \mathrm{eV}$ and is being ionized with the highest probability [see the dominant contribution of $M$-shell ionization in Fig. 2(a)]. The $O$ shell acquires hole population first and then the $N$ shell follows. This is because the pulse duration is long enough to enable continuous refilling of the created $M$ - and $N$-shell vacancies via relaxation processes. In the short-pulse case (a), however, the $M$-shell hole population emerges first and outer shells are ionized later during the pulse. In this case, the pulse duration $(0.5 \mathrm{fs})$ is shorter than the Auger lifetimes (typically a few femtoseconds), and the $M$-shell photoionization beats the Auger decays [see Fig. 9(a)], creating a multiple core-hole state. The Xe $M$ shell has 18 electrons in total. In Fig. 3(a), about $20 \%$ of the $M$-shell electrons have been ejected at the peak of the pulse, while most of the $N$ and $O$-shell electrons remain, representing the formation of a hollow atom. Here, the hollow atom implies partially empty inner shells. In fact, hollow-atom formation is known to be the key ingredient in the frustrated-absorption mechanism [11]. Since the photoionization cross section is dominated by $M$-shell ionization, removing $M$-shell electrons during the pulse reduces the effective cross section. In the present case, however, the $20 \%$ reduction of the effective Xe $M$-shell cross section is compensated by the increase of the photon flux and the increasing contribution of the outershell photoabsorptions as the pulse gets shorter. Therefore the number of absorbed photons is hardly affected as a function of the pulse duration [Fig. 2(a)]. These $M$-shell holes eventually relax and disappear after the pulse end, as shown in the right half of Fig. 3(a). At the end of the time propagation, one can see that the hole populations of the $N$ and $O$ shells for $0.5 \mathrm{fs}$ (a) are smaller than those for $500 \mathrm{fs}$ (b), i.e., the shorter pulse, the less the ionization. The average final charge is reduced from $+18(500 \mathrm{fs})$ to +16 ( $0.5 \mathrm{fs})$.

Next, in the high-fluence cases [(c) and (d)], one can see an interesting behavior of the $M$-shell hole population. For the long-pulse case (d), the $M$-shell holes are slowly populated after most of the $N$-shell and $O$-shell electrons are ionized, because no more relaxation can occur after losing most of these electrons. In this case, the nonzero population of $M$ shell holes does not represent a hollow atom. Note that the $\mathrm{Xe}$ atom has already reached a high charge state when the $M$-shell holes start to be populated. Thus the $M$-shell hole formation is limited by one-photon ionization or by resonant excitation. The one-photon $M$-shell ionization stops at +19 and the REXMI region covers the charge states from +5 to +38 (see Fig. 8). The x-ray beam parameters used in (d) yield an average final charge state of +29 . In contrast, for the short-pulse case (c), the $M$-shell holes are rapidly and strongly populated before hole formation in the $N$ and $O$ shells, since in this regime $M$-shell photoionization is much faster than Auger decay [see Fig. 9(b)]. In this case, more than half of the $M$-shell electrons are ionized during the pulse, resulting in the very efficient formation of a multiple core-hole state, i.e., a massively hollow atom. This early formation of a multiple core-hole state turns out to be the key mechanism underlying the breakdown of frustrated absorption, because it bypasses the single-photon absorption limit to reach a higher charge state. The $M$-shell hole formation at early charge states is no longer restricted by the direct one-photon ionization limit or by the REXMI mechanism. Since the fluence is sufficiently high in this case, reduction of the cross section is no longer a limiting factor. The creation of a massively hollow atom, which remains highly excited in the course of the ionization, as illustrated in Fig. 10(a), makes it possible to reach a higher charge state. The $\mathrm{x}$-ray beam parameters used in Fig. 3(c) yield an average final charge state of +35 , which is by far higher than +29 with the longer pulse duration used in Fig. 3(d). In Appendix C, we plot exemplary ionization pathways for both short and long pulse cases.

It is interesting to see, by comparing Figs. 3(c) and 3(d), that the early $M$-shell hole formation in the short-pulse case eventually causes more ionization from the $M$ shell than the late $M$-shell hole formation in the long-pulse case, even though the number of the $M$-shell photoabsorption events remains approximately constant as the pulse duration is varied [see Fig. 2(b)]. The increment of the total number of photoabsorption events for shorter pulses in Fig. 2(b) is clearly due to the increment of the outershell contribution (the difference of the solid and dashed blue lines). However, the outershell ionization by itself cannot explain how higher charge states can be reached using shorter pulses, because $N$ - and $O$-shell electrons are all removed by the end of the time evolution, irrespective of the pulse duration [see Figs. 3(c) and 3(d)], and the highest charge state is determined by the $M$-shell hole population. Note that with outershell ionization alone it is not possible go beyond a charge state of +26 , simply because all $N$ - and $O$-shell electrons are removed in the ground state of $\mathrm{Xe}^{26+}$. Therefore it is crucial to circumvent the $M$-shell absorption limit by the early and massive formation of $M$-shell holes. Then, the atom can reach the maximum charge state via excited states, with the help of an increasing number of outershell ionizations.

This concept of the early and massive formation of multiple core holes is consistent with the preservation of the innershell vacancy proposed in Ref. [34]. However, the mechanism of the "antifrustrated" absorption behavior presented here does not rely on the resonance effect, because the same phenomena persist with and without REXMI in our calculations, as demonstrated in Fig. 7 in Appendix A.

The hole population dynamics for individual shells depicted in Fig. 3 provide a discernible visualization of the concepts of "peeling" and "coring" of atoms, which were originally conceived to describe different $\mathrm{x}$-ray ionization mechanisms for different photon energies [50]. In fact, at a photon energy of $1.2 \mathrm{keV}$, x-ray multiphoton ionization is mostly initiated by coring ( $M$-shell ionization). For long pulses [(b) and (d)], core-hole states after coring have enough 

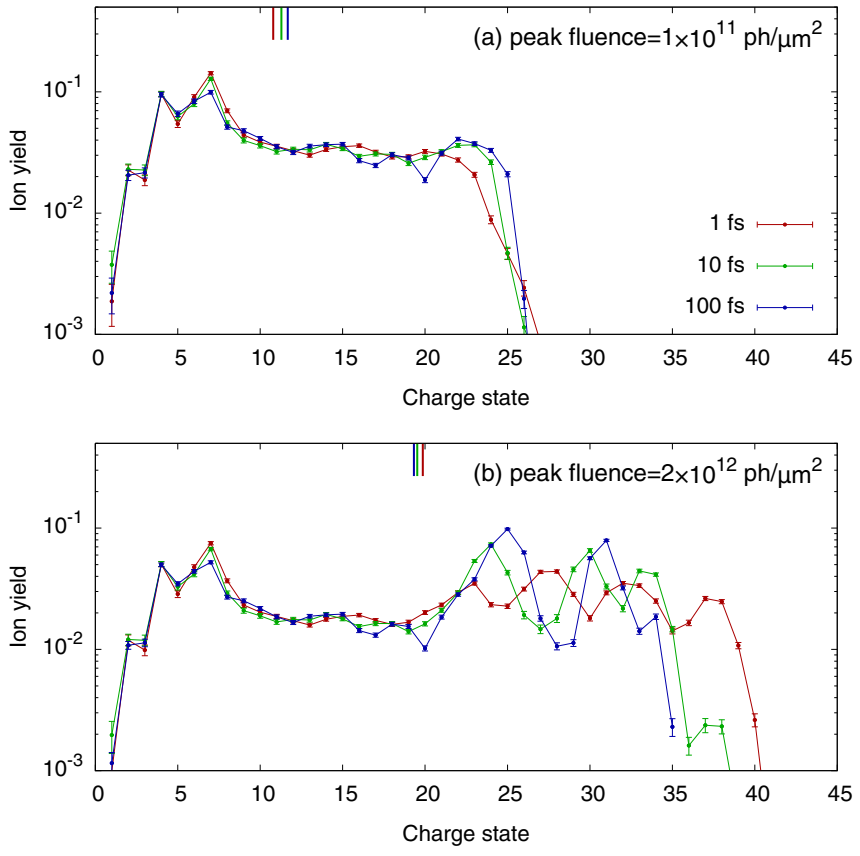

FIG. 4. Volume-integrated charge-state distributions of $\mathrm{Xe}$ at $1200 \mathrm{eV}$ for a peak fluence of (a) $1 \times 10^{11}$ and (b) $2 \times 10^{12}$ $\mathrm{ph} / \mu \mathrm{m}^{2}$. The spatial fluence distribution of a single Gaussian profile is assumed. The mean charges for corresponding pulse durations are marked with color bars at the top. The error bar indicates the statistical error.

time to fully relax, boosting outershell hole population first. Thus the hole dynamics effectively show "peeling" of atomic shells, i.e., electrons are ejected sequentially, from outer to inner shells. For short pulses [(a) and (c)], those core-hole states survive during the pulse and $M$-shell hole population emerges first and $N$ - and $O$-shell hole population follows, corresponding to the "coring" process. Early and extensive coring in (c) provides a detour for the ionization limit set by single-photon absorption, resulting in the largest degree of ionization.

\section{Volume-integrated charge-state distributions of $\mathrm{Xe}$ at $1200 \mathrm{eV}$}

In experiment, atoms interacting with an x-ray beam in different positions within the interaction volume experience different fluence values. The measured charge-state distribution (CSD), therefore, contains contributions from a range of fluence values. In order to make a quantitative comparison, theoretical CSDs corresponding to each fluence point are convolved with the experimental fluence distribution [51]. This procedure is called volume integration [11,51]. Since the pulse-duration dependence of mean charges (also CSDs) is sensitive to fluence values as demonstrated in Fig. 1 (and Fig. 7), it is not evident whether the breakdown of frustrated absorption remains significant after volume integration.

Figure 4 shows calculated CSDs after volume integration. 40 fluence points are used and each fluence point has more than 450 Monte Carlo trajectories. The spatial profile of the fluence distribution is assumed as a single Gaussian, and the maximum fluence value at the center (peak fluence) deter-
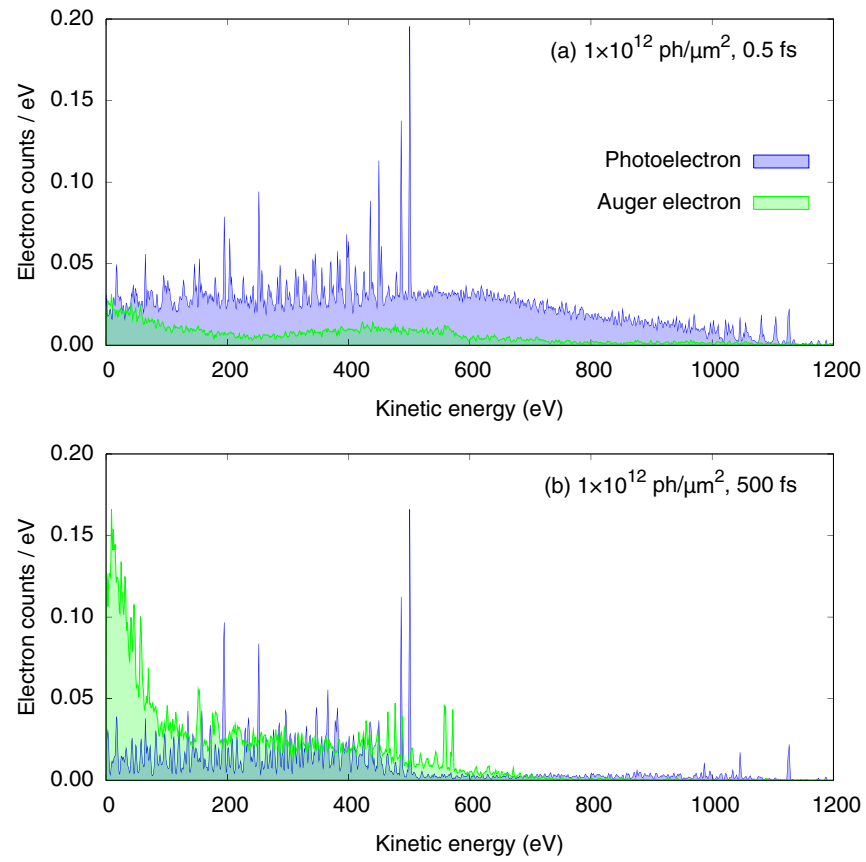

FIG. 5. Electron spectra of Xe at $1200 \mathrm{eV}$ for (a) the short-pulse case of $0.5 \mathrm{fs}$ and (b) the long-pulse case of $500 \mathrm{fs}$. For both cases, a high fluence of $1 \times 10^{12} \mathrm{ph} / \mu \mathrm{m}^{2}$ is employed. Electron counts per $\mathrm{eV}$ are convolved with a 2-eV (FWHM) Gaussian for better visibility. For clarity, photoelectrons (blue) and Auger electrons (green) are plotted with different colors.

mines the whole spatial fluence distribution. In the low-peakfluence case in Fig. 4(a), one can see the frustrated absorption behavior: almost no differences are found in the CSDs for different pulse durations, but the yields of high charge states are reduced at the shortest pulse duration $(1 \mathrm{fs})$. The mean charges, which are indicated at the top of the figure, are slightly reduced as the pulse duration decreases. In the highpeak-fluence case in Fig. 4(b), the yields of high charges are governed by interactions at high fluences. The CSDs extend to increasingly high charge states as the pulse duration becomes shorter, and their structure is sensitive to the pulse duration. Our results demonstrate that the "antifrustrated" absorption behavior at a sufficiently high peak fluence prevails in CSDs even after volume integration is applied, taking into account a broad range of fluences in the Gaussian profile. The mean charges, however, show little sensitivity to the pulse duration. These volume-integrated CSDs can be directly compared with experimental data and can thus be used for a verification of the breakdown of frustrated absorption.

\section{Electron spectra of $\mathrm{Xe}$ at $\mathbf{1 2 0 0} \mathrm{eV}$ for short and long pulses}

The different ionization mechanisms for short and long pulses at ultrahigh x-ray fluences are exhibited not only in mean charges or charge-state distributions, but also in electron spectra. Figure 5 shows photoelectron and Auger electron spectra of Xe at $1200 \mathrm{eV}$ for short (0.5 fs) and long (500 fs) pulses at a fluence of $1 \times 10^{12} \mathrm{ph} / \mu \mathrm{m}^{2}$ as used in the highfluence case of Fig. 2(b). Note that these electron spectra are not volume-integrated. As expected from Fig. 2(b), the 

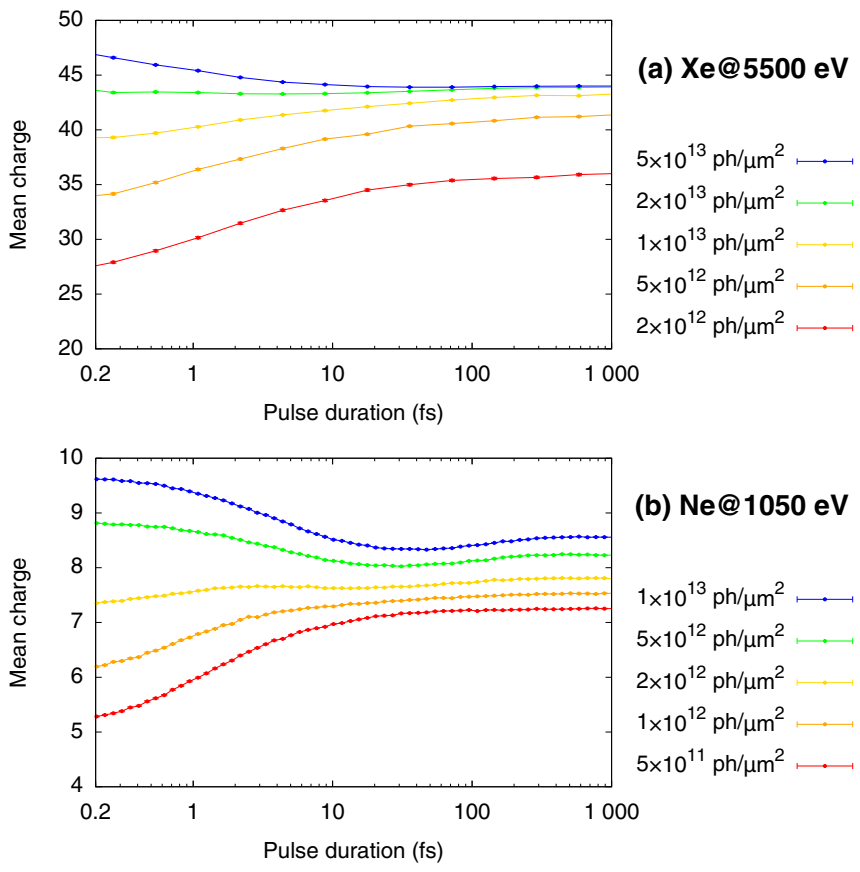

FIG. 6. Final mean charge of (a) $\mathrm{Xe}$ at $5500 \mathrm{eV}$ and (b) $\mathrm{Ne}$ at $1050 \mathrm{eV}$ as a function of pulse duration. The error bar indicates the statistical error.

total number of Auger electrons in the spectra decreases and the total number of photoelectrons increases as the pulse becomes shorter. The enhancement of photoelectrons over a broad energy range in the short-pulse case indicates that photoionization occurs in $M, N$, and $O$ shells. In particular, the emergence of high-energy $(>500 \mathrm{eV})$ photoelectrons in comparison with the long-pulse case gives a hint that $N$ - and $O$-shell ionizations are enhanced as the pulse gets shorter [see Fig. 2(b)]. Also there is a strong suppression of low-energy $(<100 \mathrm{eV})$ Auger electrons in the short-pulse case, which can be attributed to a reduction of $N-X X$ Auger channels. Thus the electron spectra can be used complementarily to the ion spectra to verify the breakdown of frustrated absorption in the experiment.

\section{E. Pulse-duration dependence of ionization dynamics of $\mathrm{Xe}$ at $5500 \mathrm{eV}$ and $\mathrm{Ne}$ at $1050 \mathrm{eV}$}

Does the breakdown of frustrated absorption happen only for heavy atoms at soft $\mathrm{x}$ rays? Our analysis of the breakdown mechanism reveals that it happens when the core-electron ionization stops at a certain charge state where the photon energy is insufficient in the ground electronic state to ionize via onephoton absorption. Figure 6(a) shows the final mean charge of $\mathrm{Xe}$ in the hard-x-ray range for several fluences. At $5.5 \mathrm{keV}$, the multiphoton multiple ionization dynamics of $\mathrm{Xe}$ is initiated by $L$-shell ionization [14,15], but this direct one-photon absorption channel closes at $\mathrm{Xe}^{22+}$. At low fluences $\left(2 \times 10^{12}\right.$ to $1 \times 10^{13} \mathrm{ph} / \mu \mathrm{m}^{2}$ ), the mean charge follows the trend expected from frustrated absorption, whereas at high fluences $\left(2 \times 10^{13}\right.$ and $\left.5 \times 10^{13} \mathrm{ph} / \mu \mathrm{m}^{2}\right)$, the mean charge increases slightly for decreasing pulse duration. Figure $6(\mathrm{~b})$ displays the final mean charge of Ne at soft $\mathrm{x}$ rays for several fluences. At
$1050 \mathrm{eV}$, the direct one-photon ionization limit is $\mathrm{Ne}^{6+}$. The formation of a hollow atom (empty $K$ shell) at charge states below +6 , facilitated by high fluence and short pulse duration, ultimately leads to charge states of +9 or +10 via further $L$-shell ionization. One can clearly see the impact of frustrated absorption at low fluences $\left(5 \times 10^{11}\right.$ to $\left.2 \times 10^{12} \mathrm{ph} / \mu \mathrm{m}^{2}\right)$ and the opposite trend at high fluences $\left(5 \times 10^{12}\right.$ and $1 \times$ $10^{13} \mathrm{ph} / \mu \mathrm{m}^{2}$ ). Note that the number of absorbed photons is actually reduced for the low-fluence cases of (a) Xe at $5.5 \mathrm{keV}$ and (b) $\mathrm{Ne}$ at $1050 \mathrm{eV}$, upholding the concept of frustrated absorption (see Appendix D).

If the photon energy is high enough to ionize all ion species that are formed during the ionization dynamics (for example, $\mathrm{Ne}$ at $2 \mathrm{keV}$ ), an intense $\mathrm{x}$-ray pulse can fully strip all electrons [11], so there is no further enhancement via multiple corehole formation for short pulses. Therefore, in the case of light atoms at hard $\mathrm{x}$ rays, we expect that the paradigm of frustrated absorption still applies.

\section{F. Towards attosecond XFEL pulses}

The latest advances in XFEL technology make it possible to generate attosecond pulse durations $[38,40]$ with tens of $\mu \mathrm{J}$ pulse energy, progressing towards terawatt-attosecond XFEL pulses [37] with a pulse energy greater than $0.1 \mathrm{~mJ}$. If the full power of $1 \mathrm{TW}$ is tightly focused to a focal size of $1 \mu \mathrm{m} \times$ $1 \mu \mathrm{m}$, the peak intensity is $10^{20} \mathrm{~W} / \mathrm{cm}^{2}$. Our present study suggests that, in the attosecond regime (0.2-1 fs) in Fig. 1, the ionization is reduced for shorter pulses with a pulse energy of $10 \mu \mathrm{J}$, assuming it is focused to $1 \mu \mathrm{m}^{2}$ (corresponding to $5 \times$ $10^{10} \mathrm{ph} / \mu \mathrm{m}^{2}$, red line in Fig. 1), while terawatt power will facilitate antifrustrated absorption (corresponding to $5 \times 10^{11}$ $\mathrm{ph} / \mu \mathrm{m}^{2}$, green, and $1 \times 10^{12} \mathrm{ph} / \mu \mathrm{m}^{2}$, blue line in Fig. 1).

When the pulse duration approaches the sub-femtosecond regime, one needs to consider two issues: the energy bandwidth and the validity of the rate-equation approach. On the one hand, with the current XFEL scheme of self-amplified spontaneous emission (SASE), the energy bandwidth is given by the $x$-ray coherence time, typically of the order of tens of attoseconds [52], which is determined by the narrowest peak within a spiky SASE pulse. If the pulse duration approaches this timescale, the spectrum becomes Fourier-limited. In our study, the energy bandwidth is $12 \mathrm{eV}$ ( $1 \%$ of the photon energy of $1.2 \mathrm{keV}$ ), corresponding to a coherence time of $\sim 0.15 \mathrm{fs}$, and the shortest pulse duration under consideration here is $0.2 \mathrm{fs}$. On the other hand, the rate-equation approach employed in the present work does not take into account temporal coherence, assuming that the timescale of photoabsorption is longer than the given coherence time. Higher fluence and shorter pulse duration enhance the photoabsorption rate, thus the photoabsorption timescale becomes shorter. Assuming the photoabsorption cross section is of the order of $\mathrm{Mb}$, the timescale of photoabsorption at a peak intensity of $1.2 \times$ $10^{18} \mathrm{~W} / \mathrm{cm}^{2}\left(1.2 \mathrm{keV}, 1 \times 10^{12} \mathrm{ph} / \mu \mathrm{m}^{2}\right.$, and $\left.15 \mathrm{fs} F W H M\right)$ becomes about $0.16 \mathrm{fs}$. If the peak intensity becomes higher, it might be necessary to consider coherence effects such as Rabi flopping [53-56]. Note that the breakdown of frustrated absorption already manifests at lower intensities (longer pulse durations at a fixed fluence), for example, pulse durations longer than 15 fs at $1 \times 10^{12} \mathrm{ph} / \mu \mathrm{m}^{2}$ (blue curve) in Fig. 1, 
corresponding to intensities lower than $1.2 \times 10^{18} \mathrm{~W} / \mathrm{cm}^{2}$, which are achievable at existing XFEL facilities.

\section{CONCLUSION}

In summary, we show that the concept of frustrated absorption, which has been established as a unique feature of the XFEL interaction with light atoms, is no longer generally valid at high $\mathrm{x}$-ray fluences. We demonstrate that the ionization suppression for shorter pulses, which superficially manifests frustrated absorption, is attributed to the suppression of Auger decays. We also find ionization enhancement for shorter pulses, i.e., antifrustrated absorption, when the x-ray fluence is sufficiently high to produce certain charge states higher than the one set by the direct one-photon ionization limit or by the REXMI limit. As the pulse duration becomes shorter, $\mathrm{x}$-ray multiphoton ionization can be either suppressed or enhanced, depending on the $\mathrm{x}$-ray fluence. Therefore, when the pulse duration is very short, particularly when approaching the attosecond regime, it is critical to consider the dependence of the ion signal not only on the $\mathrm{x}$-ray fluence but also on the $\mathrm{x}$-ray intensity. In the XUV regime, where direct multiphoton ionization is possibly dominant [57], higher yields of high charge states for shorter pulses were observed [58]. In the $\mathrm{x}$-ray regime presented here, sequential multiphoton multiple ionization is still dominant, but at ultrahigh fluence many photons can be absorbed in a quasinonsequential fashion, causing the creation of a massively hollow atom. We demonstrate that the antifrustrated absorption is due to the efficient formation of the massively hollow atom. Our finding of the breakdown of frustrated absorption at extremely high-intensity and extremely short-pulse duration is not only a new manifestation of the complexity that can arise in the interaction of a many-
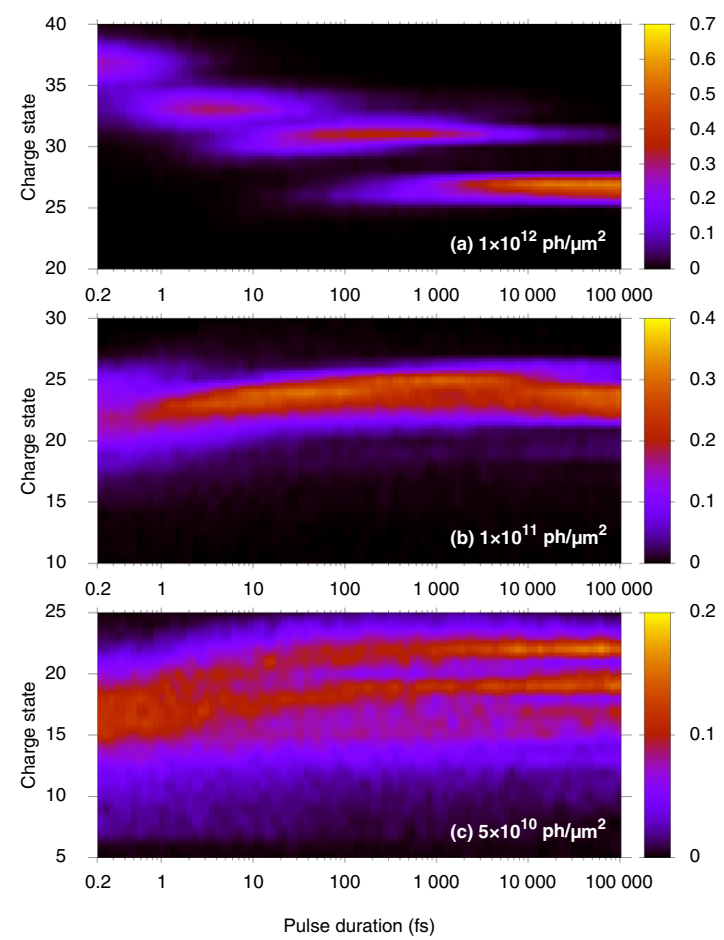

body system with high-intensity radiation, but we also expect that this phenomenon will have to be taken into account in future XFEL experiments, particularly those dedicated to utilizing upcoming terawatt-attosecond $\mathrm{x}$-ray pulses.

\section{ACKNOWLEDGMENTS}

This work was supported in part by the Cluster of Excellence "Advanced Imaging of Matter" of the Deutsche Forschungsgemeinschaft (DFG) - EXC 2056 - project ID 390715994.

\section{APPENDIX A: CHARGE-STATE DISTRIBUTION AND RESONANT EXCITATION CROSS SECTION OF Xe AT $1200 \mathrm{eV}$}

Figure 7 depicts a color map of the charge-state distribution (CSD) of Xe for three selected fluences: [(a) and (d)] $1 \times 10^{12}$, [(b) and (e) $1 \times 10^{11}$, and $[(\mathrm{c})$ and (f) $] 5 \times 10^{10} \mathrm{ph} / \mu \mathrm{m}^{2}$. The mean charges in Fig. 1 are obtained from these charge-state distributions. Thus the mean charge in Fig. 1 and the CSD in Fig. 7 provide similar information, but the CSD presents a more detailed view of the outcome of the ionization dynamics, for example, revealing resonance structures. Here, with the help of CSDs, we will focus on the impact of REXMI $[13,32]$ on frustrated absorption. In the left panels, (a)-(c), resonant excitations are included, while in the right panels, (d)-(f), they are excluded in the underlying calculations. If REXMI is possible, it dominates the ionization dynamics because of the large cross sections of resonant excitation processes. Although REXMI is the dominant process for the high-charge formation in Fig. 1, it does not explain why the paradigm of frustrated absorption fails at high fluences, simply because the same

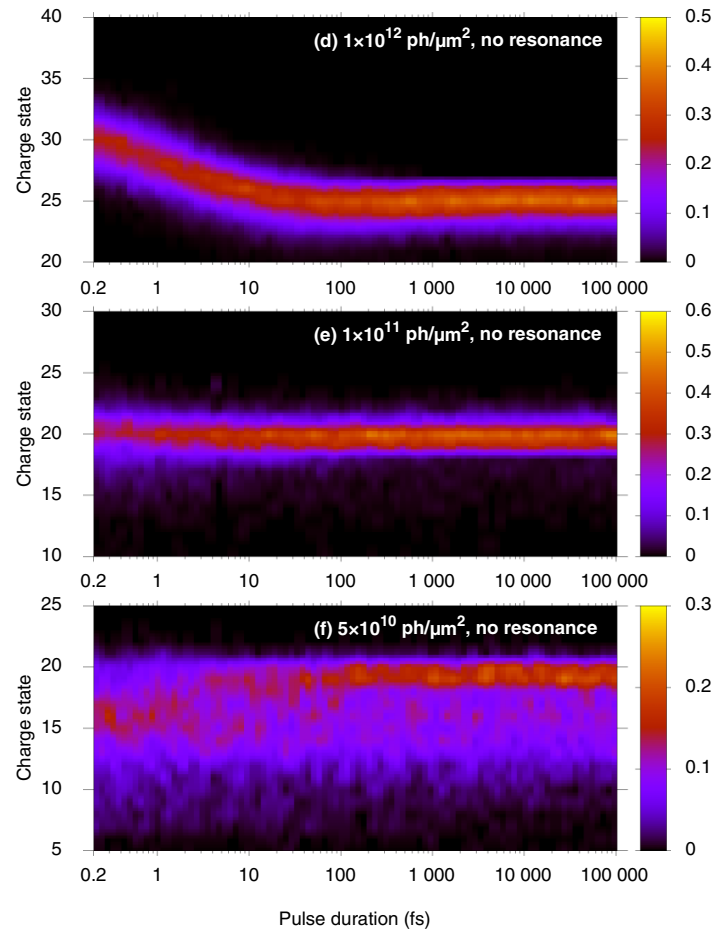

FIG. 7. Color map of the charge-state distribution of $\mathrm{Xe}$ at $1200 \mathrm{eV}$ as a function of pulse duration. 


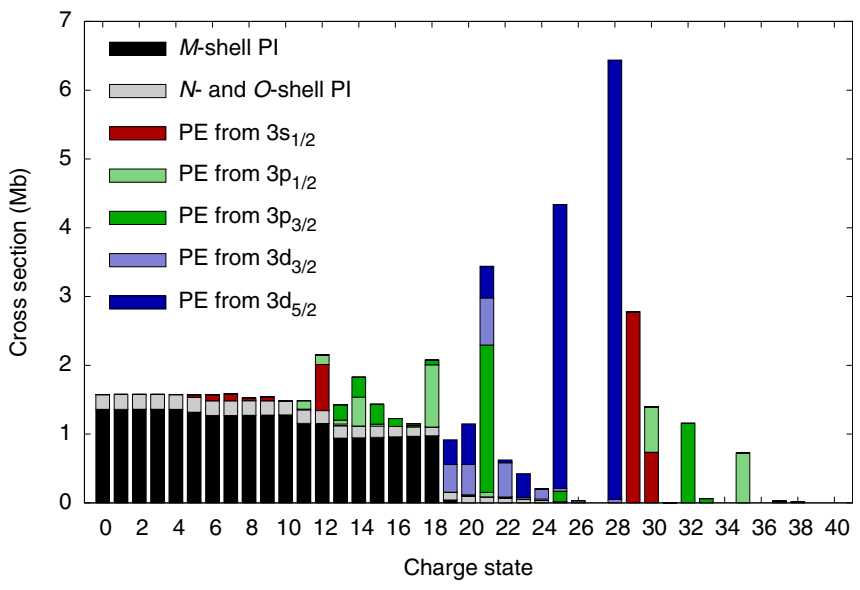

FIG. 8. Calculated cross sections for $M-, N$-, and $O$-shell photoionization (PI) and resonant $M$-shell photoexcitation (PE) of Xe at $1200 \mathrm{eV}$ and $1 \%$ bandwidth. Different red, green and blue colors indicate from which subshell the resonant excitation occurs.

trends are observed in the CSDs calculated with and without REXMI. As shown in Fig. 7, for both cases (with and without resonances), higher charge states are generated for shorter pulse durations at high fluence, whereas the yield of high charge states decreases as the pulse duration decreases at low fluence.

For the resonance cases, (a)-(c), more structures are exhibited, which are features of specific resonant excitations in REXMI. The discrete fringes above +25 in Fig. 7(a) can be analyzed with cross section calculations. Figure 8 shows photoionization and resonant photoexcitation cross sections of ground-state $\mathrm{Xe}$ ions at $1200 \mathrm{eV}$ and $1 \%$ bandwidth. Table I lists where the discrete fringes appear in Fig. 7(a) and how they are assigned to specific transitions based on the cross section contributions in Fig. 8. One can see that the peak position at $+Q$ in the cross section in Fig. 8 roughly corresponds to the peak at $+(Q+1)$ in the CSD in Fig. 7, as demonstrated in Ref. [15].

\section{APPENDIX B: COMPETITION BETWEEN PHOTOIONIZATION AND AUGER DECAY OF Xe AT 1200 eV}

Figure 9 compares the photoionization rates and the Auger rates of multiple core-hole states of Xe at $1200 \mathrm{eV}$. The

TABLE I. Transition assignment of fringes in charge-state distributions. The CSDs are shown in Fig. 7(a) and the cross sections are in Fig. 8.

\begin{tabular}{lcc}
\hline \hline Maxima in CSDs & Maxima in cross sections & Transition \\
\hline around +27 & at +25 & $3 d_{5 / 2} \rightarrow 6 f_{7 / 2}$ \\
around +31 & at +28 & $3 d_{5 / 2} \rightarrow 5 f_{7 / 2}$ \\
& at +29 & $3 s_{1 / 2} \rightarrow 4 p_{3 / 2}$ \\
& at +30 & $3 s_{1 / 2} \rightarrow 4 p_{1 / 2}$ \\
around +33 & & $3 p_{1 / 2} \rightarrow 4 d_{3 / 2}$ \\
around +37 & at +32 & $3 p_{3 / 2} \rightarrow 4 d_{5 / 2}$ \\
\hline \hline
\end{tabular}
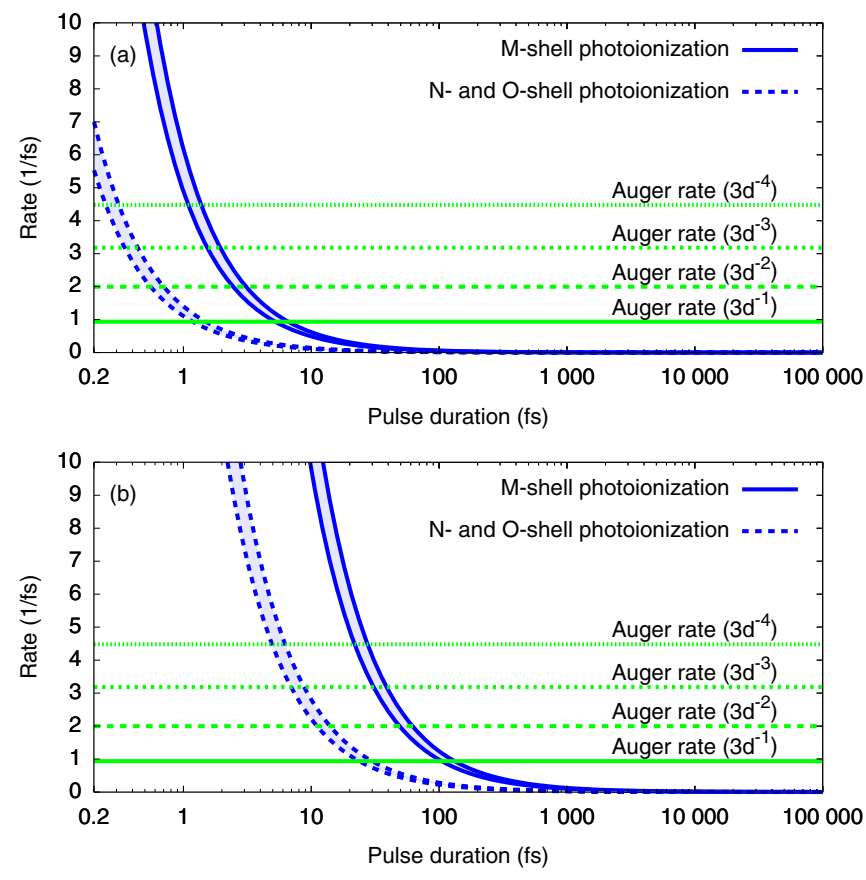

FIG. 9. Comparison between the Auger rates and the photoionization rates of $\mathrm{Xe}$ at $1200 \mathrm{eV}$ at the peak of the pulse as a function of pulse duration: (a) low fluence $\left(5 \times 10^{10} \mathrm{ph} / \mu \mathrm{m}^{2}\right)$ and (b) high fluence $\left(1 \times 10^{12} \mathrm{ph} / \mu \mathrm{m}^{2}\right)$.

$M$-shell photoionization rates are depicted by a range enclosed by two solid blue lines, corresponding to initial-state configurations $3 \mathrm{~d}^{-1}$ and $3 \mathrm{~d}^{-4}$, respectively, and the outershell ( $N$ - and $O$-shell) photoionization rates lie in the area between the two dashed blue lines (also corresponding to the initial-state configurations $3 \mathrm{~d}^{-1}$ and $3 \mathrm{~d}^{-4}$ ). In the low-fluence case of $5 \times 10^{10} \mathrm{ph} / \mu \mathrm{m}^{2}(\mathrm{a})$, the $M$-shell photoionization rate exceeds the Auger rate of the single core-hole state when the pulse duration is less than $\sim 7 \mathrm{fs}$, and the outershell ( $N$ - and $O$-shell) photoionization rates become higher than the Auger rate for the pulse duration less than $\sim 1$ fs. The fact that $N$ and $O$-shell ionization proceeds faster than the Auger decay for the very short pulses is responsible for the suppression of the Auger processes depicted in Fig. 2(a), because fewer electrons remain available for the decay. In the high-fluence case of $1 \times 10^{12} \mathrm{ph} / \mu \mathrm{m}^{2}(\mathrm{~b})$, the photoionization rates of all the shells surpass the Auger decays of single to quadruple core-hole states, when the pulse duration approaches a few femtoseconds. This enables the absorption of several photons before any relaxation processes, creating a massively hollow atom as illustrated in the ionization pathway in Fig. 10(a), where 13 photons are absorbed and $9 M$-shell electrons are removed in a 0.5 -fs pulse before the first Auger decay (for more details see also Appendix C).

In Fig. 9, the photoionization rate is calculated from the cross section times the photon flux. The photon flux at the peak of a Gaussian-shaped pulse is given by $J=$ $\sqrt{4 \ln 2 / \pi}(F / \tau)$, where $F$ is the fluence and $\tau$ is the pulse duration (FWHM). The calculated partial cross sections of Xe $\left(3 \mathrm{~d}^{-1}\right)$ at $1200 \mathrm{eV}$ for the $M, N$, and $O$ shells are $\sigma_{M}=1.3$ $\mathrm{Mb}, \sigma_{N}=0.22 \mathrm{Mb}$, and $\sigma_{O}=0.014 \mathrm{Mb}$, respectively. For Xe $\left(3 \mathrm{~d}^{-4}\right), \sigma_{M}=1.0 \mathrm{Mb}, \sigma_{N}=0.27 \mathrm{Mb}$, and $\sigma_{O}=0.023 \mathrm{Mb}$. 


\section{APPENDIX C: IONIZATION PATHWAY OF Xe AT $1200 \mathrm{eV}$}

In Fig. 10, we plot the total energy of a $\mathrm{Xe}$ atom for an exemplary ionization pathway, illustrating the ionization mechanism at a photon energy of $1200 \mathrm{eV}$. We compare two different pulse durations, (a) 0.5 and (b) $500 \mathrm{fs}$, at the same ultrahigh fluence of $1 \times 10^{12} \mathrm{ph} / \mu \mathrm{m}^{2}$. The energy of neutral $\mathrm{Xe}$ is set to zero, and the black line is the groundconfiguration energy for given charge states. A series of arrows shows a pathway of sequential ionization dynamics. Different colors indicate different processes (blue: photoionization, red: photoexcitation, green: Auger decay, and yellow: fluorescence). Different thicknesses represent which shells are involved in the corresponding process (thickest: $M$-shell photoionization/excitation, $M-X X$ Auger decay, or $M-X$ fluorescence; medium: $N$-shell photoionization or $N-X X$ Auger decay; and thinnest: photoionization from $O$ shell or above). The black triangle marker indicates the peak of the x-ray pulse, and some accompanying timescales are displayed. At the end of each pathway, fluorescence decays occur on timescales of picoseconds (not clearly visible in these plots).

In the short-pulse case [Fig. 10(a)], one can see a series of photoionizations and photoexcitations with only a few Auger decays, bringing the system up to a $\sim 10-\mathrm{keV}$ excited state. This detouring channel via excited states makes it possible to reach a higher charge state than expected based on the singlephoton absorption limit. In this high-fluence and short-pulse case, it is most likely that both $M$-shell and outershell $(N$ and $O$-shell) photoionization rates exceed the Auger rates [see $\tau=0.5$ fs in Fig. 9(b), where $\tau$ is the pulse duration]. Thus
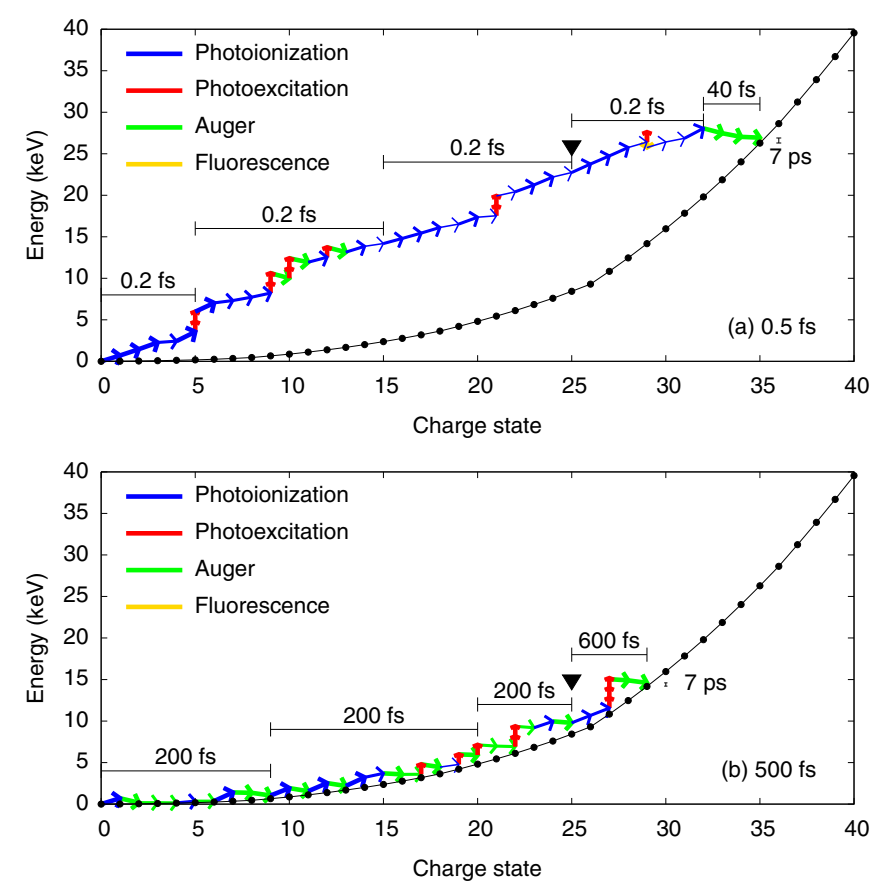

FIG. 10. Comparison of the ionization mechanisms of Xe at $1200 \mathrm{eV}$ for (a) short (0.5 fs) and (b) long (500 fs) pulse durations. A fluence of $1 \times 10^{12} \mathrm{ph} / \mu \mathrm{m}^{2}$ is used for both cases. Shown are exemplary, typical ionization pathways for each case. The black circles represent the electronic ground-state energy as a function of the atomic charge state. the outershell photoionizations (thin arrows) remove $N$ - and $O$-shell electrons that are required for Auger decays, reducing the number of Auger decays as shown in Fig. 2(b). In contrast, in the long-pulse case [Fig. 10(b)], the electronic configurations visited in the course of the ionization dynamics remain close (on the keV scale of Fig. 10) to the ionization pathway via ground electronic configurations (black circles). In this case, the photoionization rates are smaller than the Auger rates [see $\tau=500$ fs in Fig. 9(b)]. After one photoionization, the resulting core-hole state relaxes almost immediately via a series of Auger decays, which prevents the formation of a highly energetic hollow atom.

\section{APPENDIX D: SIGNATURE OF FRUSTRATED ABSORPTION FOR Xe AT $5500 \mathrm{eV}$ AND Ne AT $1050 \mathrm{eV}$}

Figure 11 shows the number of absorbed photons in (a) Xe at $5500 \mathrm{eV}$ and (b) $\mathrm{Ne}$ at $1050 \mathrm{eV}$, corresponding to Fig. 6. At low fluences (orange and red), the number of photoabsorption events is reduced when the pulse duration becomes shorter, which is responsible for the decrease of the mean charge in Fig. 6, together with the reduction of the number of Auger decays (not shown). This illustrates that the concept of frustrated absorption is valid in both cases. At high fluences (green and blue), the number of photoabsorption events increases for shorter pulses, manifesting the breakdown of frustrated absorption. Note that, at an intermediate fluence (yellow), for both $\mathrm{Xe}$ at $5500 \mathrm{eV}$ and $\mathrm{Ne}$ at $1050 \mathrm{eV}$, the number of photoabsorptions increases as the pulse gets shorter, even though the mean charge decreases (see Fig. 6). In this case, the reduced number of Auger decays is responsible for the suppression of the mean charge for shorter pulses.
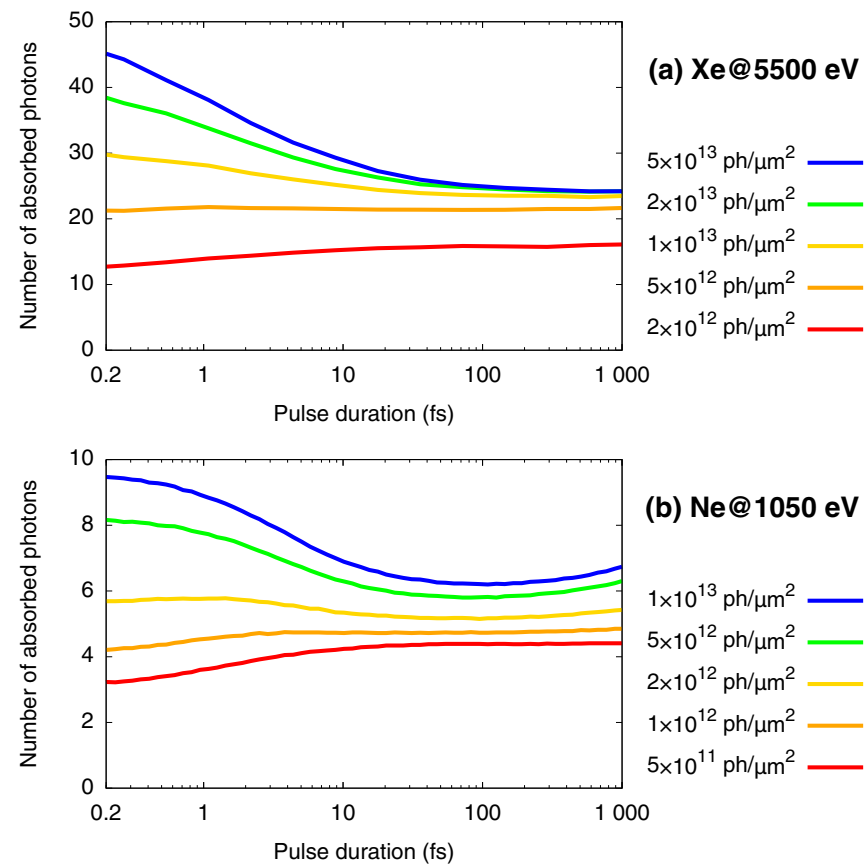

FIG. 11. Number of absorbed photons in (a) $\mathrm{Xe}$ at $5500 \mathrm{eV}$ and (b) $\mathrm{Ne}$ at $1050 \mathrm{eV}$ as a function of pulse duration. 
[1] P. Emma, R. Akre, J. Arthur, R. Bionta, C. Bostedt, J. Bozek, A. Brachmann, P. Bucksbaum, R. Coffee, F.-J. Decker, Y. Ding, D. Dowell, S. Edstrom, A. Fisher, J. Frisch, S. Gilevich, J. Hastings, G. Hays, P. Hering, Z. Huang, R. Iverson, H. Loos, M. Messerschmidt, A. Miahnahri, S. Moeller, H.-D. Nuhn, G. Pile, D. Ratner, J. Rzepiela, D. Schultz, T. Smith, P. Stefan, H. Tompkins, J. Turner, J. Welch, W. White, J. Wu, G. Yocky, and J. Galayda, First lasing and operation of an ångstromwavelength free-electron laser, Nat. Photon. 4, 641 (2010).

[2] T. Ishikawa, H. Aoyagi, T. Asaka, Y. Asano, N. Azumi, T. Bizen, H. Ego, K. Fukami, T. Fukui, Y. Furukawa, S. Goto, H. Hanaki, T. Hara, T. Hasegawa, T. Hatsui, A. Higashiya, T. Hirono, N. Hosoda, M. Ishii, T. Inagaki, Y. Inubushi, T. Itoga, Y. Joti, M. Kago, T. Kameshima, H. Kimura, Y. Kirihara, A. Kiyomichi, T. Kobayashi, C. Kondo, T. Kudo, H. Maesaka, X. M. Maréchal, T. Masuda, S. Matsubara, T. Matsumoto, T. Matsushita, S. Matsui, M. Nagasono, N. Nariyama, H. Ohashi, T. Ohata, T. Ohshima, S. Ono, Y. Otake, C. Saji, T. Sakurai, T. Sato, K. Sawada, T. Seike, K. Shirasawa, T. Sugimoto, S. Suzuki, S. Takahashi, H. Takebe, K. Takeshita, K. Tamasaku, H. Tanaka, R. Tanaka, T. Tanaka, T. Togashi, K. Togawa, A. Tokuhisa, H. Tomizawa, K. Tono, S. Wu, M. Yabashi, M. Yamaga, A. Yamashita, K. Yanagida, C. Zhang, T. Shintake, H. Kitamura, and N. Kumagai, A compact x-ray free-electron laser emitting in the sub-ångström region, Nat. Photonics 6, 540 (2012).

[3] H.-S. Kang, C.-K. Min, H. Heo, C. Kim, H. Yang, G. Kim, I. Nam, S. Y. Baek, H.-J. Choi, G. Mun, B. R. Park, Y. J. Suh, D. C. Shin, J. Hu, J. Hong, S. Jung, S.-H. Kim, K. Kim, D. Na, S. S. Park, Y. J. Park, J.-H. Han, Y. G. Jung, S. H. Jeong, H. G. Lee, S. Lee, S. Lee, W.-W. Lee, B. Oh, H. S. Suh, Y. W. Parc, S.-J. Park, M. H. Kim, N.-S. Jung, Y.-C. Kim, M.-S. Lee, B.-H. Lee, C.-W. Sung, I.-S. Mok, J.-M. Yang, C.-S. Lee, H. Shin, J. H. Kim, Y. Kim, J. H. Lee, S.-Y. Park, J. Kim, J. Park, I. Eom, S. Rah, S. Kim, K. H. Nam, J. Park, J. Park, S. Kim, S. Kwon, S. H. Park, K. S. Kim, H. Hyun, S. N. Kim, S. Kim, S.-m. Hwang, M. J. Kim, C.-y. Lim, C.-J. Yu, B.-S. Kim, T.-H. Kang, K.-W. Kim, S.-H. Kim, H.-S. Lee, H.-S. Lee, K.-H. Park, T.-Y. Koo, D.-E. Kim, and I. S. Ko, Hard X-ray free-electron laser with femtosecond-scale timing jitter, Nat. Photonics 11, 708 (2017).

[4] T. Tschentscher, C. Bressler, J. Grünert, A. Madsen, A. P. Mancuso, M. Meyer, A. Scherz, H. Sinn, and U. Zastrau, Photon beam transport and scientific instruments at the European XFEL, Appl. Sci. 7, 592 (2017).

[5] C. J. Milne, T. Schietinger, M. Aiba, A. Alarcon, J. Alex, A. Anghel, V. Arsov, C. Beard, P. Beaud, S. Bettoni, M. Bopp, H. Brands, M. Brönnimann, I. Brunnenkant, M. Calvi, A. Citterio, P. Craievich, M. Csatari Divall, M. Dällenbach, M. D'Amico, A. Dax, Y. Deng, A. Dietrich, R. Dinapoli, E. Divall, S. Dordevic, S. Ebner, C. Erny, H. Fitze, U. Flechsig, R. Follath, F. Frei, F. Gärtner, R. Ganter, T. Garvey, Z. Geng, I. Gorgisyan, C. Gough, A. Hauff, C. P. Hauri, N. Hiller, T. Humar, S. Hunziker, G. Ingold, R. Ischebeck, M. Janousch, P. Juranić, M. Jurcevic, M. Kaiser, B. Kalantari, R. Kalt, B. Keil, C. Kittel, G. Knopp, W. Koprek, H. T. Lemke, T. Lippuner, D. Llorente Sancho, F. Löhl, C. Lopez-Cuenca, F. Märki, F. Marcellini, G. Marinkovic, I. Martiel, R. Menzel, A. Mozzanica, K. Nass, G. L. Orlandi, C. Ozkan Loch, E. Panepucci, M. Paraliev, B. Patterson, B. Pedrini, M. Pedrozzi, P. Pollet, C. Pradervand, E. Prat, P. Radi,
J.-Y. Raguin, S. Redford, J. Rehanek, J. Réhault, S. Reiche, M. Ringele, J. Rittmann, L. Rivkin, A. Romann, M. Ruat, C. Ruder, L. Sala, L. Schebacher, T. Schilcher, V. Schlott, T. Schmidt, B. Schmitt, X. Shi, M. Stadler, L. Stingelin, W. Sturzenegger, J. Szlachetko, D. Thattil, D. M. Treyer, A. Trisorio, W. Tron, S. Vetter, C. Vicario, D. Voulot, M. Wang, T. Zamofing, C. Zellweger, R. Zennaro, E. Zimoch, R. Abela, L. Patthey, and H.-H. Braun, SwissFEL: The Swiss X-ray free electron laser, Appl. Sci. 7, 720 (2017).

[6] J. P. Marangos, Introduction to the new science with x-ray free electron lasers, Contemp. Phys. 52, 551 (2011).

[7] C. Bostedt, S. Boutet, D. M. Fritz, Z. Huang, H. J. Lee, H. T. Lemke, A. Robert, W. F. Schlotter, J. J. Turner, and G. J. Williams, Linac coherent light source: The first five years, Rev. Mod. Phys. 88, 015007 (2016).

[8] E. A. Seddon, J. A. Clarke, D. J. Dunning, C. Masciovecchio, C. J. Milne, F. Parmigiani, D. Rugg, J. C. H. Spence, N. R. Thompson, K. Ueda, S. M. Vinko, J. S. Wark, and W. Wurth, Short-wavelength free-electron laser sources and science: a review, Rep. Prog. Phys. 80, 115901 (2017).

[9] J. C. H. Spence, XFELs for structure and dynamics in biology, IUCrJ 4, 322 (2017)

[10] L. Young, K. Ueda, M. Gühr, P. H. Bucksbaum, M. Simon, S. Mukamel, N. Rohringer, K. C. Prince, C. Masciovecchio, M. Meyer, A. Rudenko, D. Rolles, C. Bostedt, M. Fuchs, D. A. Reis, R. Santra, H. Kapteyn, M. Murnane, H. Ibrahim, F. Légaré, M. Vrakking, M. Isinger, D. Kroon, M. Gisselbrecht, A. L'Huillier, H. J. Wörner, and S. R. Leone, Roadmap of ultrafast X-ray atomic and molecular physics, J. Phys. B: At. Mol. Opt. Phys. 51, 032003 (2018).

[11] L. Young, E. P. Kanter, B. Krässig, Y. Li, A. M. March, S. T. Pratt, R. Santra, S. H. Southworth, N. Rohringer, L. F. DiMauro, G. Doumy, C. A. Roedig, N. Berrah, L. Fang, M. Hoener, P. H. Bucksbaum, J. P. Cryan, S. Ghimire, J. M. Glownia, D. A. Reis, J. D. Bozek, C. Bostedt, and M. Messerschmidt, Femtosecond electronic response of atoms to ultra-intense x-rays, Nature (London) 466, 56 (2010).

[12] G. Doumy, C. Roedig, S.-K. Son, C. I. Blaga, A. D. DiChiara, R. Santra, N. Berrah, C. Bostedt, J. D. Bozek, P. H. Bucksbaum, J. P. Cryan, L. Fang, S. Ghimire, J. M. Glownia, M. Hoener, E. P. Kanter, B. Krässig, M. Kuebel, M. Messerschmidt, G. G. Paulus, D. A. Reis, N. Rohringer, L. Young, P. Agostini, and L. F. DiMauro, Nonlinear Atomic Response to Intense Ultrashort X Rays, Phys. Rev. Lett. 106, 083002 (2011).

[13] B. Rudek, S.-K. Son, L. Foucar, S. W. Epp, B. Erk, R. Hartmann, M. Adolph, R. Andritschke, A. Aquila, N. Berrah, C. Bostedt, J. Bozek, N. Coppola, F. Filsinger, H. Gorke, T. Gorkhover, H. Graafsma, L. Gumprecht, A. Hartmann, G. Hauser, S. Herrmann, H. Hirsemann, P. Holl, A. Hömke, L. Journel, C. Kaiser, N. Kimmel, F. Krasniqi, K.-U. Kühnel, M. Matysek, M. Messerschmidt, D. Miesner, T. Möller, R. Moshammer, K. Nagaya, B. Nilsson, G. Potdevin, D. Pietschner, C. Reich, D. Rupp, G. Schaller, I. Schlichting, C. Schmidt, F. Schopper, S. Schorb, C.-D. Schröter, J. Schulz, M. Simon, H. Soltau, L. Strüder, K. Ueda, G. Weidenspointner, R. Santra, J. Ullrich, A. Rudenko, and D. Rolles, Ultra-efficient ionization of heavy atoms by intense x-ray free-electron laser pulses, Nat. Photonics 6, 858 (2012).

[14] H. Fukuzawa, S.-K. Son, K. Motomura, S. Mondal, K. Nagaya, S. Wada, X.-J. Liu, R. Feifel, T. Tachibana, Y. Ito, M. Kimura, 
T. Sakai, K. Matsunami, H. Hayashita, J. Kajikawa, P. Johnsson, M. Siano, E. Kukk, B. Rudek, B. Erk, L. Foucar, E. Robert, C. Miron, K. Tono, Y. Inubushi, T. Hatsui, M. Yabashi, M. Yao, R. Santra, and K. Ueda, Deep Inner-Shell Multiphoton Ionization by Intense X-Ray Free-Electron Laser Pulses, Phys. Rev. Lett. 110, 173005 (2013).

[15] B. Rudek, K. Toyota, L. Foucar, B. Erk, R. Boll, C. Bomme, J. Correa, S. Carron, S. Boutet, G. J. Williams, K. R. Ferguson, R. Alonso-Mori, J. E. Koglin, T. Gorkhover, M. Bucher, C. S. Lehmann, B. Krässig, S. H. Southworth, L. Young, C. Bostedt, K. Ueda, T. Marchenko, M. Simon, Z. Jurek, R. Santra, A. Rudenko, S.-K. Son, and D. Rolles, Relativistic and resonant effects in the ionization of heavy atoms by ultra-intense hard x-rays, Nat. Commun. 9, 4200 (2018).

[16] M. Hoener, L. Fang, O. Kornilov, O. Gessner, S. T. Pratt, M. Gühr, E. P. Kanter, C. Blaga, C. Bostedt, J. D. Bozek, P. H. Bucksbaum, C. Buth, M. Chen, R. Coffee, J. Cryan, L. DiMauro, M. Glownia, E. Hosler, E. Kukk, S. R. Leone, B. McFarland, M. Messerschmidt, B. Murphy, V. Petrovic, D. Rolles, and N. Berrah, Ultraintense X-Ray Induced Ionization, Dissociation, and Frustrated Absorption in Molecular Nitrogen, Phys. Rev. Lett. 104, 253002 (2010).

[17] L. Fang, M. Hoener, O. Gessner, F. Tarantelli, S. T. Pratt, O. Kornilov, C. Buth, M. Gühr, E. P. Kanter, C. Bostedt, J. D. Bozek, P. H. Bucksbaum, M. Chen, R. Coffee, J. Cryan, M. Glownia, E. Kukk, S. R. Leone, and N. Berrah, Double CoreHole Production in $\mathrm{N}_{2}$ : Beating the Auger Clock, Phys. Rev. Lett. 105, 083005 (2010).

[18] B. Erk, D. Rolles, L. Foucar, B. Rudek, S. W. Epp, M. Cryle, C. Bostedt, S. Schorb, J. Bozek, A. Rouzee, A. Hundertmark, T. Marchenko, M. Simon, F. Filsinger, L. Christensen, S. De, S. Trippel, J. Küpper, H. Stapelfeldt, S. Wada, K. Ueda, M. Swiggers, M. Messerschmidt, C. D. Schröter, R. Moshammer, I. Schlichting, J. Ullrich, and A. Rudenko, Ultrafast Charge Rearrangement and Nuclear Dynamics Upon Inner-Shell Multiple Ionization of Small Polyatomic Molecules, Phys. Rev. Lett. 110, 053003 (2013)

[19] B. Erk, D. Rolles, L. Foucar, B. Rudek, S. W. Epp, M. Cryle, C. Bostedt, S. Schorb, J. Bozek, A. Rouzee, A. Hundertmark, T. Marchenko, M. Simon, F. Filsinger, L. Christensen, S. De, S. Trippel, J. Küpper, H. Stapelfeldt, S. Wada, K. Ueda, M. Swiggers, M. Messerschmidt, C. D. Schröter, R. Moshammer, I. Schlichting, J. Ullrich, and A. Rudenko, Inner-shell multiple ionization of polyatomic molecules with an intense $\mathrm{x}$-ray freeelectron laser studied by coincident ion momentum imaging, J. Phys. B: At. Mol. Opt. Phys. 46, 164031 (2013).

[20] B. Erk, R. Boll, S. Trippel, D. Anielski, L. Foucar, B. Rudek, S. W. Epp, R. Coffee, S. Carron, S. Schorb, K. R. Ferguson, M. Swiggers, J. D. Bozek, M. Simon, T. Marchenko, J. Küpper, I. Schlichting, J. Ullrich, C. Bostedt, D. Rolles, and A. Rudenko, Imaging charge transfer in iodomethane upon x-ray photoabsorption, Science 345, 288 (2014).

[21] K. Motomura, E. Kukk, H. Fukuzawa, S.-I. Wada, K. Nagaya, S. Ohmura, S. Mondal, T. Tachibana, Y. Ito, R. Koga, T. Sakai, K. Matsunami, A. Rudenko, C. Nicolas, X.-J. Liu, C. Miron, Y. Zhang, Y. Jiang, J. Chen, M. Anand, D. E. Kim, K. Tono, M. Yabashi, M. Yao, and K. Ueda, Charge and nuclear dynamics induced by deep inner-shell multiphoton ionization of $\mathrm{CH}_{3} \mathrm{I}$ molecules by intense $\mathrm{x}$-ray free-electron laser pulses, J. Phys. Chem. Lett. 6, 2944 (2015).
[22] R. Boll, B. Erk, R. Coffee, S. Trippel, T. Kierspel, C. Bomme, J. D. Bozek, M. Burkett, S. Carron, K. R. Ferguson, L. Foucar, J. Küpper, T. Marchenko, C. Miron, M. Patanen, T. Osipov, S. Schorb, M. Simon, M. Swiggers, S. Techert, K. Ueda, C. Bostedt, D. Rolles, and A. Rudenko, Charge transfer in dissociating iodomethane and fluoromethane molecules ionized by intense femtosecond x-ray pulses, Struct. Dyn. 3, 043207 (2016).

[23] A. Rudenko, L. Inhester, K. Hanasaki, X. Li, S. J. Robatjazi, B. Erk, R. Boll, K. Toyota, Y. Hao, O. Vendrell, C. Bomme, E. Savelyev, B. Rudek, L. Foucar, S. H. Southworth, C. S. Lehmann, B. Krässig, T. Marchenko, M. Simon, K. Ueda, K. R. Ferguson, M. Bucher, T. Gorkhover, S. Carron, R. Alonso-Mori, J. E. Koglin, J. Correa, G. J. Williams, S. Boutet, L. Young, C. Bostedt, S.-K. Son, R. Santra, and D. Rolles, Femtosecond response of polyatomic molecules to ultra-intense hard x-rays, Nature (London) 546, 129 (2017).

[24] M. Protopapas, C. H. Keitel, and P. L. Knight, Atomic physics with super-high intensity lasers, Rep. Prog. Phys. 60, 389 (1997).

[25] N. Rohringer and R. Santra, X-ray nonlinear optical processes using a self-amplified spontaneous emission free-electron laser, Phys. Rev. A 76, 033416 (2007).

[26] H. Winter and F. Aumayr, Hollow atoms, J. Phys. B: At. Mol. Opt. Phys. 32, R39 (1999).

[27] B. D. Patterson, Crystallography using an x-ray free-electron laser, Crystallogr. Rev. 20, 242 (2014).

[28] J. Miao, T. Ishikawa, I. K. Robinson, and M. M. Murnane, Beyond crystallography: Diffractive imaging using coherent X-ray light sources, Science 348, 530 (2015).

[29] B. Ziaja, Z. Jurek, N. Medvedev, V. Saxena, S.-K. Son, and R. Santra, Towards realistic simulations of macromolecules irradiated under the conditions of coherent diffraction imaging with an X-ray free-electron laser, Photonics 2, 256 (2015).

[30] S. M. Vinko, O. Ciricosta, B. I. Cho, K. Engelhorn, H.-K. Chung, C. R. D. Brown, T. Burian, J. Chalupský, R. W. Falcone, C. Graves, V. Hájková, A. Higginbotham, L. Juha, J. Krzywinski, H. J. Lee, M. Messerschmidt, C. D. Murphy, Y. Ping, A. Scherz, W. Schlotter, S. Toleikis, J. J. Turner, L. Vysin, T. Wang, B. Wu, U. Zastrau, D. Zhu, R. W. Lee, P. A. Heimann, B. Nagler, and J. S. Wark, Creation and diagnosis of a solid-density plasma with an x-ray free-electron laser, Nature (London) 482 , 59 (2012).

[31] K. Toyota, S.-K. Son, and R. Santra, Interplay between relativistic energy corrections and resonant excitations in $\mathrm{x}$-ray multiphoton ionization dynamics of Xe atoms, Phys. Rev. A 95, 043412 (2017).

[32] B. Rudek, D. Rolles, S.-K. Son, L. Foucar, B. Erk, S. Epp, R. Boll, D. Anielski, C. Bostedt, S. Schorb, R. Coffee, J Bozek, S. Trippel, T. Marchenko, M. Simon, L. Christensen, S. De, S.-I. Wada, K. Ueda, I. Schlichting, R. Santra, J. Ullrich, and A. Rudenko, Resonance-enhanced multiple ionization of krypton at an X-ray free-electron laser, Phys. Rev. A 87, 023413 (2013).

[33] P. J. Ho, C. Bostedt, S. Schorb, and L. Young, Theoretical Tracking of Resonance-Enhanced Multiple Ionization Pathways in X-Ray Free-Electron Laser Pulses, Phys. Rev. Lett. 113, 253001 (2014).

[34] P. J. Ho, E. P. Kanter, and L. Young, Resonance-mediated atomic ionization dynamics induced by ultraintense $\mathrm{x}$-ray pulses, Phys. Rev. A 92, 063430 (2015). 
[35] K. Motomura, H. Fukuzawa, S.-K. Son, S. Mondal, T. Tachibana, Y. Ito, M. Kimura, K. Nagaya, T. Sakai, K. Matsunami, S. Wada, H. Hayashita, J. Kajikawa, X.-J. Liu, R. Feifel, P. Johnsson, M. Siano, E. Kukk, B. Rudek, B. Erk, L. Foucar, E. Robert, C. Miron, K. Tono, Y. Inubushi, T. Hatsui, M. Yabashi, M. Yao, R. Santra, and K. Ueda, Sequential multiphoton multiple ionization of atomic argon and xenon irradiated by x-ray free-electron laser pulses at SACLA, J. Phys. B: At. Mol. Opt. Phys. 46, 164024 (2013).

[36] H. Mimura, H. Yumoto, S. Matsuyama, T. Koyama, K. Tono, Y. Inubushi, T. Togashi, T. Sato, J. Kim, R. Fukui, Y. Sano, M. Yabashi, H. Ohashi, T. Ishikawa, and K. Yamauchi, Generation of $10^{20} \mathrm{~W} \mathrm{~cm}^{-2}$ hard X-ray laser pulses with two-stage reflective focusing system, Nat. Commun. 5, 3539 (2014).

[37] E. Prat and S. Reiche, Simple Method to Generate TerawattAttosecond X-Ray Free-Electron-Laser Pulses, Phys. Rev. Lett. 114, 244801 (2015).

[38] S. Huang, Y. Ding, Y. Feng, E. Hemsing, Z. Huang, J. Krzywinski, A. A. Lutman, A. Marinelli, T. J. Maxwell, and D. Zhu, Generating Single-Spike Hard X-Ray Pulses with Nonlinear Bunch Compression in Free-Electron Lasers, Phys. Rev. Lett. 119, 154801 (2017).

[39] S. Serkez, G. Geloni, S. Tomin, G. Feng, E. V. Gryzlova, A. N. Grum-Grzhimailo, and M. Meyer, Overview of options for generating high-brightness attosecond $\mathrm{x}$-ray pulses at freeelectron lasers and applications at the European XFEL, J. Opt. 20, 024005 (2018).

[40] N. Hartmann, G. Hartmann, R. Heider, M. S. Wagner, M. Ilchen, J. Buck, A. O. Lindahl, C. Benko, J. Grünert, J. Krzywinski, J. Liu, A. A. Lutman, A. Marinelli, T. Maxwell, A. A. Miahnahri, S. P. Moeller, M. Planas, J. Robinson, A. K. Kazansky, N. M. Kabachnik, J. Viefhaus, T. Feurer, R. Kienberger, R. N. Coffee, and W. Helml, Attosecond time-energy structure of x-ray free-electron laser pulses, Nat. Photonics 12, 215 (2018).

[41] J. Duris, S. Li, T. Driver, E. G. Champenois, J. P. MacArthur, A. A. Lutman, Z. Zhang, P. Rosenberger, J. W. Aldrich, R. Coffee, G. Coslovich, F.-J. Decker, J. M. Glownia, G. Hartmann, W. Helml, A. Kamalov, J. Knurr, J. Krzywinski, M.-F. Lin, J. P. Marangos, M. Nantel, A. Natan, J. T. O'Neal, N. Shivaram, P. Walter, A. L. Wang, J. J. Welch, T. J. A. Wolf, J. Z. Xu, M. F. Kling, P. H. Bucksbaum, A. Zholents, Z. Huang, J. P. Cryan, and A. Marinelli, Tunable isolated attosecond X-ray pulses with gigawatt peak power from a free-electron laser, Nat. Photonics 14, 30 (2020).

[42] S.-K. Son, L. Young, and R. Santra, Impact of hollow-atom formation on coherent $\mathrm{x}$-ray scattering at high intensity, Phys. Rev. A 83, 033402 (2011).

[43] Z. Jurek, S.-K. Son, B. Ziaja, and R. Santra, XMDYN and XATOM: versatile simulation tools for quantitative modeling of x-ray free-electron laser induced dynamics of matter, J. Appl. Cryst. 49, 1048 (2016).
[44] J. M. Schäfer, L. Inhester, S.-K. Son, R. F. Fink, and R. Santra, Electron and fluorescence spectra of a water molecule irradiated by an X-ray free-electron laser pulse, Phys. Rev. A 97, 053415 (2018).

[45] H. I. B. Banks, A. Hadjipittas, and A. Emmanouilidou, Pulse dependence of prevalent pathways in xenon driven by an x-ray free-electron-laser pulse, arXiv:1908.08728.

[46] P. Lambropoulos and X. Tang, Multiple excitation and ionization of atoms by strong lasers, J. Opt. Soc. Am. B 4, 821 (1987).

[47] A. L'Huillier, L. A. Lompre, G. Mainfray, and C. Manus, Multiply charged ions induced by multiphoton absorption in rare gases at $0.53 \mu \mathrm{m}$, Phys. Rev. A 27, 2503 (1983).

[48] B. Walker, B. Sheehy, L. F. DiMauro, P. Agostini, K. J. Schafer, and K. C. Kulander, Precision Measurement of Strong Field Double Ionization Of Helium, Phys. Rev. Lett. 73, 1227 (1994).

[49] S. F. J. Larochelle, A. Talebpour, and S. L. Chin, Non-sequential multiple ionization of rare gas atoms in a Ti:Sapphire laser field, J. Phys. B: At. Mol. Opt. Phys. 31, 1201 (1998).

[50] J. Wark, X-ray laser peels and cores atoms, Nature (London) 466, 35 (2010).

[51] K. Toyota, Z. Jurek, S.-K. Son, H. Fukuzawa, K. Ueda, N. Berrah, B. Rudek, D. Rolles, A. Rudenko, and R. Santra, xcalib: a focal spot calibrator for intense $\mathrm{x}$-ray free-electron laser pulses based on the charge state distributions of light atoms, J. Synchrotron Radiat. 26, 1017 (2019).

[52] S.-K. Son and R. Santra, Monte Carlo calculation of ion, electron, and photon spectra of xenon atoms in x-ray free-electron laser pulses, Phys. Rev. A 85, 063415 (2012).

[53] E. P. Kanter, B. Krässig, Y. Li, A. M. March, P. Ho, N. Rohringer, R. Santra, S. H. Southworth, L. F. DiMauro, G. Doumy, C. A. Roedig, N. Berrah, L. Fang, M. Hoener, P. H. Bucksbaum, S. Ghimire, D. A. Reis, J. D. Bozek, C. Bostedt, M. Messerschmidt, and L. Young, Unveiling and Driving Hidden Resonances with High-Fluence, High-Intensity X-Ray Pulses, Phys. Rev. Lett. 107, 233001 (2011).

[54] N. Rohringer and R. Santra, Resonant Auger effect at high x-ray intensity, Phys. Rev. A 77, 053404 (2008).

[55] N. Rohringer and R. Santra, Strongly driven resonant Auger effect treated by an open-quantum-system approach, Phys. Rev. A 86, 043434 (2012).

[56] Y. Li, C. Gao, W. Dong, J. Zeng, Z. Zhao, and J. Yuan, Coherence and resonance effects in the ultra-intense laser-induced ultrafast response of complex atoms, Sci. Rep. 6, 18529 (2016).

[57] M. G. Makris, P. Lambropoulos, and A. Mihelič, Theory of Multiphoton Multielectron Ionization of Xenon Under Strong 93-eV Radiation, Phys. Rev. Lett. 102, 033002 (2009).

[58] N. Gerken, S. Klumpp, A. A. Sorokin, K. Tiedtke, M. Richter, V. Bürk, K. Mertens, P. Juranić, and M. Martins, TimeDependent Multiphoton Ionization of Xenon in the Soft-X-Ray Regime, Phys. Rev. Lett. 112, 213002 (2014). 